\title{
Entrapped Stent Delivery Catheter Shaft After High Risk TAVI: Retrieval \& Lessons Learned
}

\author{
Safwan Kassas, MD ${ }^{1 *}$, Peter Fattal, MD², Manoj Sharma, MD ${ }^{3}$ \\ 1 Structural cardiology, Michigan Cardiovascular Institute, Ascension ST Mary's of Michigan, Saginaw, MI, USA \\ ${ }^{2}$ Cardiac Imaging, Michigan Cardiovascular Institute, Ascension ST Mary's of Michigan, Saginaw, MI, United States \\ ${ }^{3}$ Cardiology Division, Covenant Health Care, Saginaw, MI, United States
}

\begin{abstract}
This case report will discuss a first reported complication of very high coronary occlusion risk related to valve-in-valve (VIV) balloon expandable transcatheter aortic valve replacement (TAVR). As a protective measure, an undeployed coronary stent was placed in the left anterior descending (LAD) artery. During the transcatheter heart valve (THV) deployment, the shaft of the left coronary stent catheter was firmly entrapped between the surgical valve posts and the balloon expandable TAVR frame. This prohibited the retrieval and movement of the coronary stent catheter. There were two subsequent ruptures and detachments of the coronary stent catheter while attempting to retrieve the catheter. This report will provide measures to help avoid this entrapment using VIV/balloon expandable TAVR procedures. Steps taken to successfully manage this complication will be discussed.

Copyright @ 2019 Science International Corp.
\end{abstract}

Key Words

Aortic stenosis - Retrieval of foreign body - Coronary occlusion

\section{Introduction}

Transcather aortic valve replacement for a deteriorating surgical valve in the aortic position is an approved therapy for both the self-expanding Medtronic and the balloon expandable EDWARDS valves. Coronary occlusion is a recognized complication of the TAVR procedure with a slightly higher incidence in the VIV procedures (as cited in Hamid et al., 2015). One of the protective strategies in high coronary occlusion riskTAVR is to place a coronary an undeployed stent in the coronary artery. If coronary occlusion complication occurs, the stent catheter can be pulled back and the left main or right coronary artery (RCA) ostium can be stented. The stent is extended out to reestablish coronary flow. This bail out strategy seems simple and catastrophic complications can take place, as in this case being reported.

\section{Methods}

A 63-year-old female patient with the following risk factors: hypertension, type II diabetes mellitus, dyslipidemia, and age. This patient does have a nor-

* Corresponding Author:

Safwan Kassas, MD Inteventional Cardiology Michigan Cardiovascular Institute

1015 S Washington Ave, Saginaw, MI 48601, USA

Tel. +1 248219 2481; Fax: +1 248792 7749; E-Mail: safwankassasmd@aol.com \\ (c) 2019 Journal of Structural Heart Disease \\ Published by Science International Corp. \\ http://structuralheartdisease.org/
}




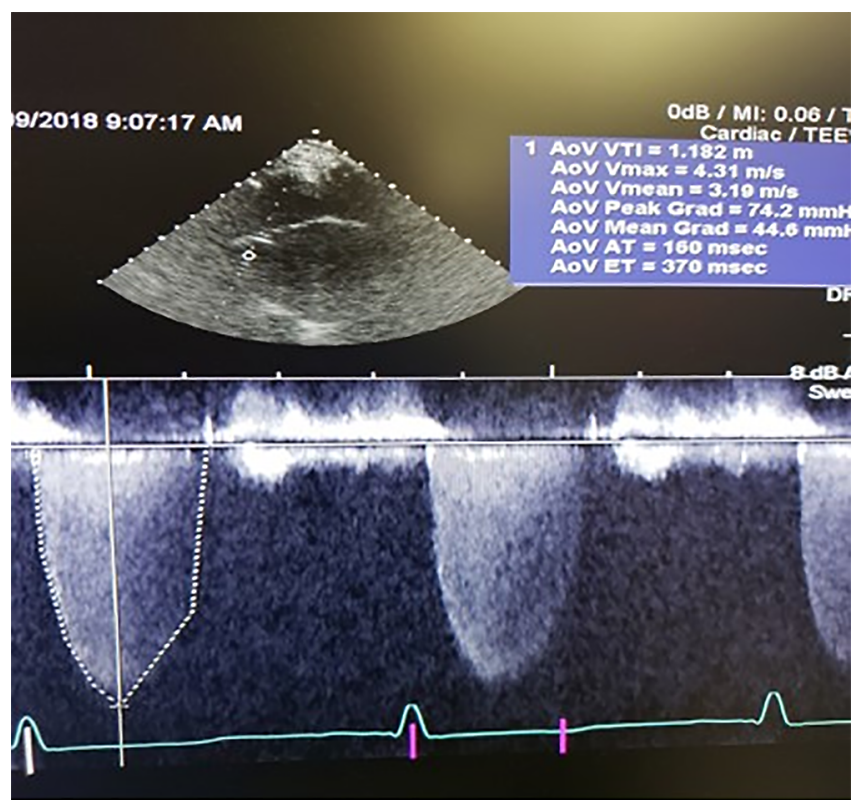

Figure 1. Mean gradient across the bioprosthetic aortic valve of $44 \mathrm{~mm} \mathrm{Hg}$ indicating severe stenotic process

mal left ventricular ejection fraction (EF) with atrial fibrillation and history of moderate mitral and tricuspid valve regurgitation. This patient has prior cardiac

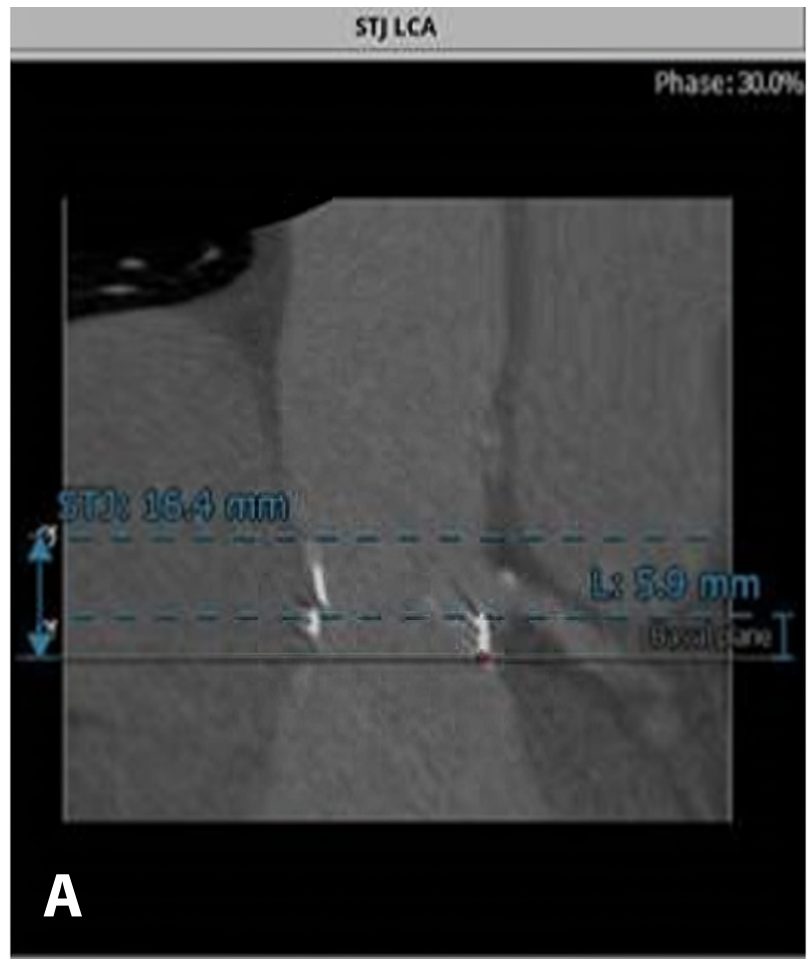

history of coronary artery disease (CAD) with a LAD stent placed in 2013. In 2011, a 25 mm Carpentier-Edwards Magna pericardial valve was used as an aortic valve replacement. Other past medical history includes: severe chronic obstructive pulmonary disease (COPD) with FEV1 of $0.7 \mathrm{~L}$ at $40 \%$ predicted, significant renal insufficiency with creatine around $2.0 \mathrm{mg} /$ $\mathrm{dL}$, glomerular filtration rate (GFR) of $32 \mathrm{~mL} /$ minute and gastrointestinal bleed with baseline hemoglobin between 8.0 and $9.0 \mathrm{~g} / \mathrm{dL}$.

This patient complained of exertional dyspnea with frequent hospital admissions due to congestive heart failure. This was associated with progressive stenotic process in the aortic valve prosthesis with the last mean gradient across the aortic valve prothesis noted at $44 \mathrm{mmHg}$ (Figure 1). A patient prosthesis mismatch was ruled out due to the mean gradient of $6 \mathrm{mmHg}$ a year post-operatively.

The heart valve team met with this patient to discuss the option of redoing the surgical aortic valve replacement or the transcatheter valve implantation. This case was risk stratified by two cardiac surgeons as a high surgical risk due the calculated STS score of

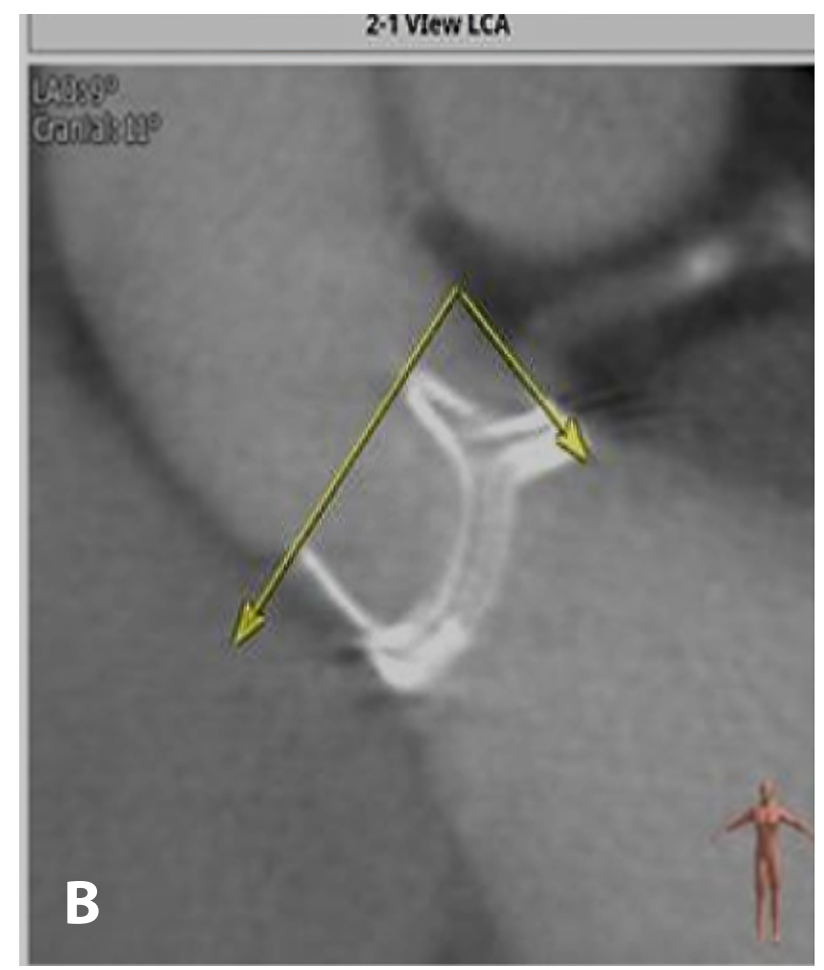

Figure 2. High coronary occlusion risk. Panel A. Left main coronary ostial height $5.9 \mathrm{~mm}$. Panel B. Surgical frame posts $17 \mathrm{~mm}$ slightly higher than STJ $16.7 \mathrm{~mm}$. 

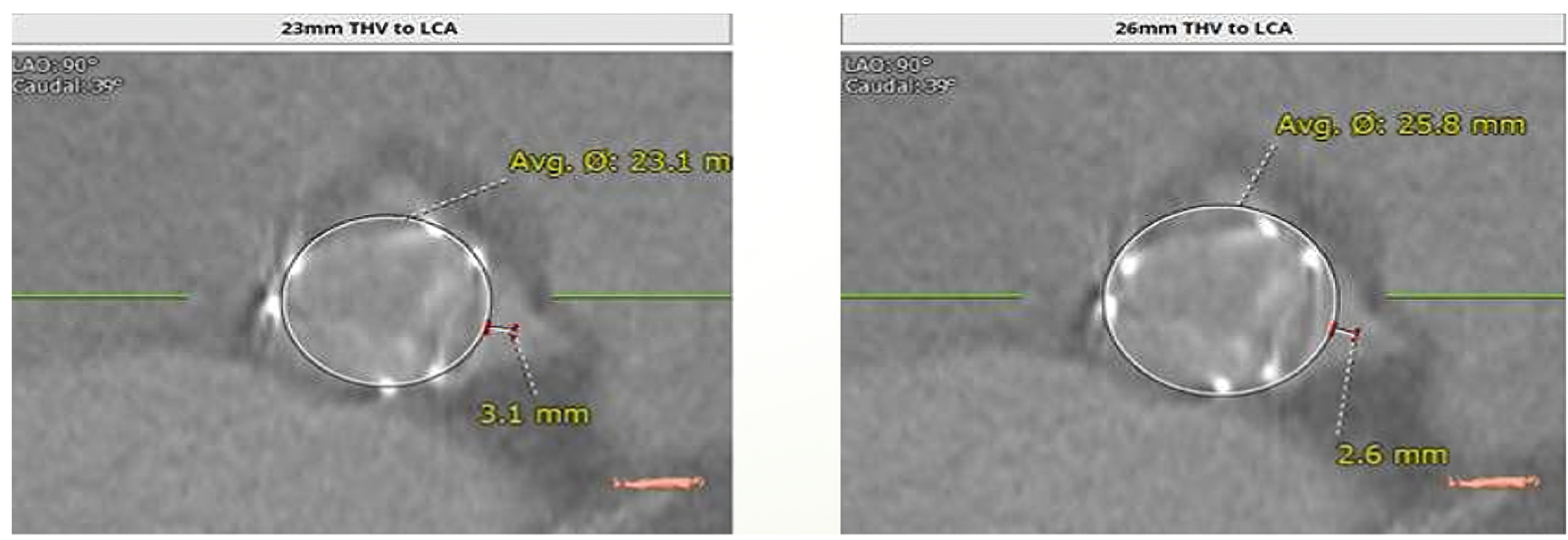

Figure 3. High coronary occlusion risk: Virtual THV coronary distance $3.1 \mathrm{~mm}$ for Edwards $23 \mathrm{~mm}$ S3 valve and $2.6 \mathrm{~mm}$ for Edwards $26 \mathrm{~mm}$ valve.

9.6. Another challenge was possibility of the patient declining to undergo redo cardiac surgery. The patient was evaluated for the transcatheter approach. A computed tomography (CT) scan was performed to evaluate the patient's suitability for the VIV procedure and evaluate procedural risks, including coronary occlusion (as cited in Dvir et al., 2015).

The manufacturer profile for the patient's $25 \mathrm{~mm}$ Carpentier-Edwards Magna pericardial valve provides a true diameter of $23 \mathrm{~mm}$ and posts elevation of 17 $\mathrm{mm}$. The true identifier for the patient's aortic valve prothesis as measured by CT scan was $21.7 \mathrm{~mm}$ by $23.9 \mathrm{~mm}$ with an average of $22.8 \mathrm{~mm}$. The sinotubular junction height was $16.4 \mathrm{~mm}$, which was slightly lower than the surgical valve posts. The average diameter was $28.4 \mathrm{~mm}$. The left main coronary ostial height was low at $5.9 \mathrm{~mm}$ and the right coronary ostial height was also low at $8.5 \mathrm{~mm}$ (Figure 2).

Both the VIV application and the CT evaluation suggested a $26 \mathrm{~mm}$ expandable Edwards SAPIEN 3 balloon and transcatheter valve or a $26 \mathrm{~mm}$ self-expanding CoreValve. These are the two valves available in the United States.

There is concern about the future difficulty accessing the low coronary arteries with very low ostial height in this patient. The sinus of Valsalva is narrow, while the sinotubular junction height is both low and narrow as compared to the surgical posts height. This made the balloon expandable EDWARDS SAPIEN 3 valve more desirable. However, the patient was at very high risk for both left main and right coronary artery occlusions (as cited in Dvir et al., 2015). High risk criteria in this case includes: first, the VTC distance (virtual THV to coronary distance), as the virtual THV ring to the left main coronary ostia. The distance was $2.6 \mathrm{~mm}$ for a $26 \mathrm{~mm}$ EDWARDS SAPIEN3 valve and a $3.1 \mathrm{~mm}$ for a $23 \mathrm{~mm}$ SAPIEN3 valve (Figure 1). Second, a slightly tilted surgical prosthesis compared to the aortic root long axis. Third, the surgical frame posts are higher than the sinotubular junction at $17 \mathrm{~mm}$ versus $16.4 \mathrm{~mm}$. Fourth, a very low coronary ostial height (Figure 3). Fifth, very small sinuses and stenotic valve pathology (Figure 3 ).

Coronary occlusion risk was discussed with the patient and surgical options were readdressed. However, the patient again declined the redo high risk surgical approach. The patient was offered a transfer to an institution. This institution is capable of splitting the aortic valve surgical prosthesis leaflets to reduce the risk of coronary occlusion (BASILICA procedure) (as cited in Khan et al., 2018). This option was declined due to family circumstances related to travel. This resulted in the patient consenting to the high risk coronary occlusion valve-in-valve TAVR. The following strategies were planned to perform this high coronary occlusion risk valve-in-valve TAVR case. Strategy one is undersizing by using a smaller THV valve at $23 \mathrm{~mm}$ rather than $26 \mathrm{~mm}$. Strategy two is using a slightly lower deployment. Strategy three is placing coronary stents in the mid LAD and mid RCA prophylactically as a bail out option if coronary occlusion or compromised flow occurs. 


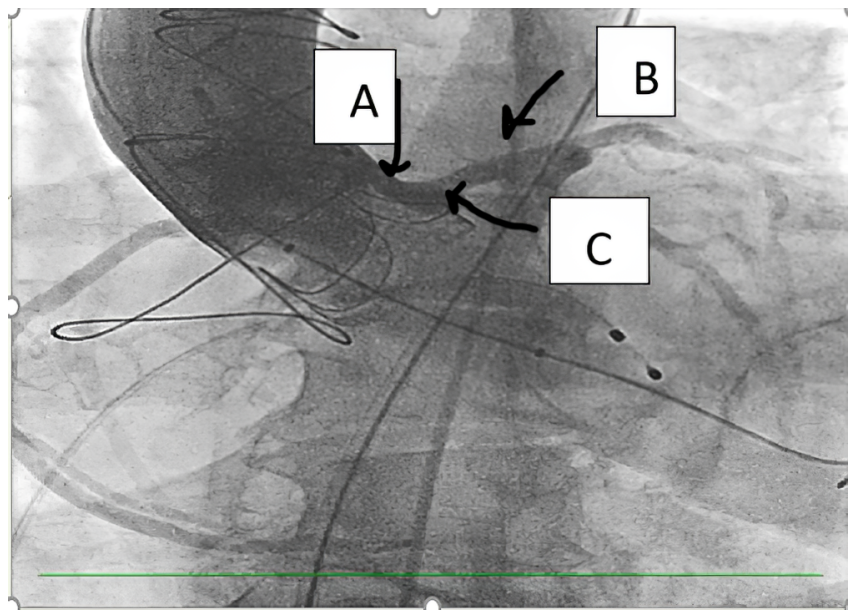

Figure 4. High coronary occlusion risk: Angiographically observed short VTC distance between left main ostium and surgical post. (A) Surgical frame post, (B) Left main artery, (C) Short left VTC distance.

The first strategy was selected. The plan was to use a $23 \mathrm{~mm}$ EDWARDS SAPIEN 3 balloon expandable valve instead of the $26 \mathrm{~mm}$ valve as suggested by the VIV application and CT analysis. Subsequently, this decision was selected due to the slightly longer VTC distance with a $23 \mathrm{~mm}$ valve to lower risk of coronary occlusion. Therefore, a $3.1 \mathrm{~mm}$ VTC distance was used versus the $2.6 \mathrm{~mm}$. Doing this created less lateral displacement of the surgical posts with a smaller diameter THV valve. A shorter frame height valve of $23 \mathrm{~mm}$ was used instead of the $26 \mathrm{~mm}$ SAPIEN 3 valve. The sinotubular junction height in this patient is $16.4 \mathrm{~mm}$, which is why the slightly shorter surgical frame post of $17 \mathrm{~mm}$ was selected. This would hypothetically cause less interaction of the surgical posts with the sinotubular junction having a shorter THV frame.

Slightly lower deployment was planned to minimize any potential interaction between the laterally displaced surgical posts and the sinotubular junction because of deployment of the THV valve. The left 2:1, right 2:1 and 1:1:1 fluoroscopic angles were identified and angiographic images were obtained. While using the XB 3.5 and JR4 guide catheters, coronary stents were placed in the mid LAD and mid RCA. There was a high likelihood a left main stent would need to be deployed to create a chimney path next to the THV stent frame. Aortic root angiography during a $23 \mathrm{~mm}$ balloon inflation in the left 2:1 angulation and the

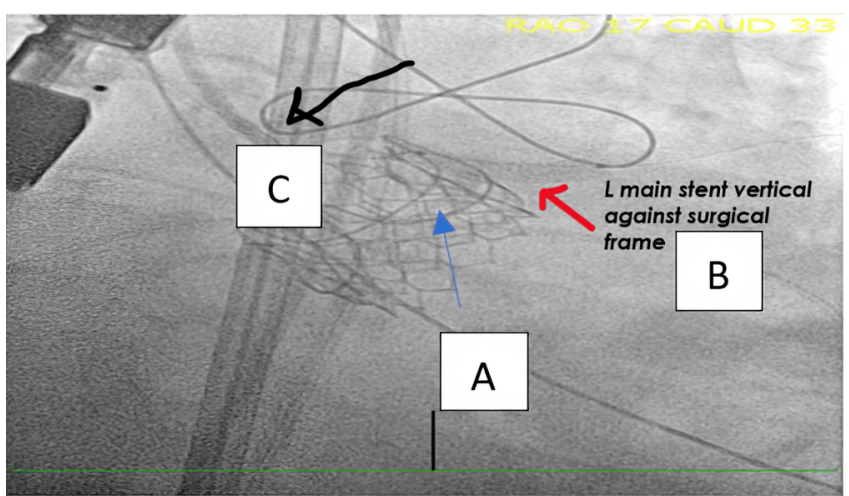

Figure 5. Coronary stent in a vertical trajectory against surgical aortic prothesis with the stent balloon can not be withdrawn back due to catheter entrapment. (A) THV. surgical prothesis frames. (B) Left main stent in a vertical trajectory against surgical bioprosthesis with deflated balloon entrapped inside the stent. (C) XB 3.5 guide catheter.

right 2:1 angulation confirmed the high coronary occlusion risk. Thrombolysis in myocardial infarction (TIMI) 3 flow was still maintained in both the left main and RCA during balloon inflation (Figure 4).

The $23 \mathrm{~mm}$ EDWARDS SAPIEN 3 valve was deployed in the left 2:1 view to evaluate the interaction between the left main ostium and the surgical valve prothesis during the THV valve deployment. Towards the end of the THV inflation period an interaction took place between the left coronary stent catheter shaft and the surgical posts/THV frame. Angiography post THV deployment showed the patient continued to have TIMI 3 flow in both the left and right coronary arteries. The wire and stent in the RCA were removed since there was no concern about RCA flow. However, due to the very short distance between the left main ostium and the surgical posts, deployment of the left coronary stent in and out of the left main coronary artery was performed. This created a vertical chimney appearance with a stented path extending to the level of the sinotubular junction. Easy future access could now be obtained if needed to the very low left main coronary artery, which had shallow sinuses and a short VTC distance of $3.1 \mathrm{~mm}$.

Significant resistance was encountered while attempting to pull the stent catheter back from the parked mid LAD segment. While positioning the stent at the left main level, the stent catheter shaft was en- 

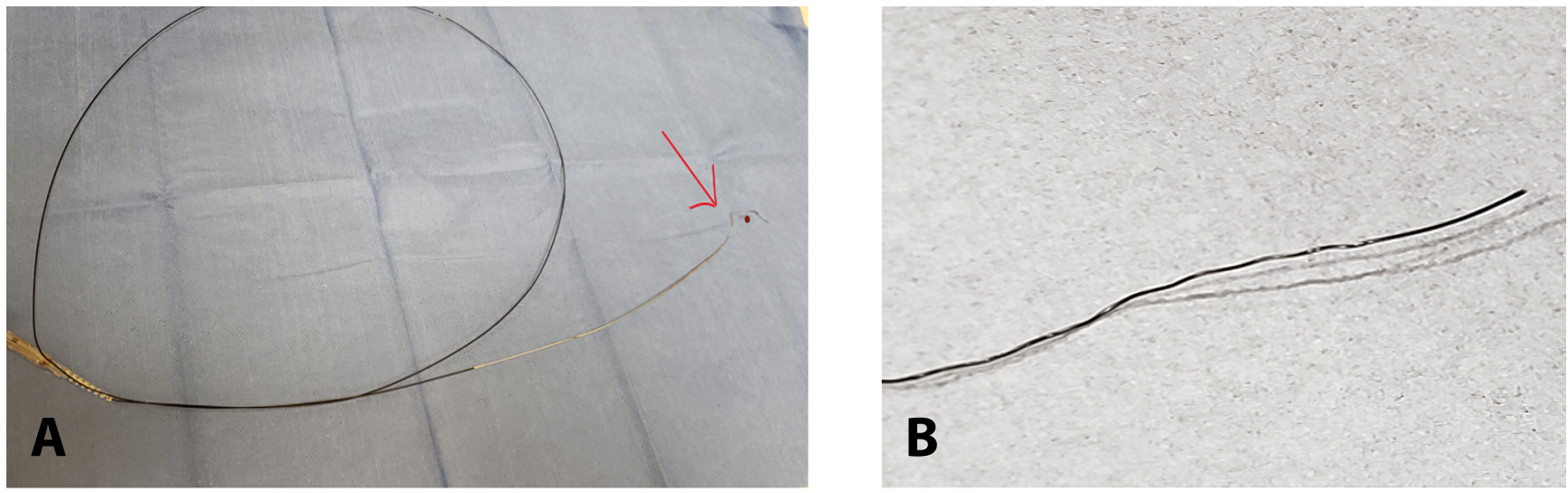

Figure 6. Panel A. First rupture of stent catheter shaft at monorail point. Panel B. Second rupture of catheter shaft because of snaring.

trapped firmly between the surgical valve posts and the EDWARDS frame. This prohibited the ability to retrieve the catheter. The stent was retrieved while using a mechanical and forceful pull where the coronary stent struts were in contact with the surgical valve/THV frames. No further adjustment forward or backwards was feasible. Therefore, the chimney path that with the intent to be created was unable to be retrieved by the stent catheter. The only available option to proceed was to deploy the left coronary stent in an undesirable position. The stent was partially in the left main artery and extended out, contacting in a vertical trajectory to the surgical/THV frames (Figure 5).

The plan was to be able to drag the stent balloon from its entrapped course between the surgical posts and the SAPIEN 3 frame and remove from the body. The left main coronary artery was rewired through the struts of the existing stent. Balloon dilatation of the left main stent strut was then performed. A second stent was deployed, creating a parallel stented path that was initially felt to be necessary. Since the deflated balloon was unable to be retrieved, the winged balloon failed to pass in-between the surgical post and the SAPIEN 3 THV frame. A manual pulling force was applied to facilitate the retrieval of the stent balloon passing in-between the surgical bioprosthesis post and the EDWARDS SAPIEN 3 frame passage. Unfortunately, the balloon catheter shaft ruptured at the monorail port because of the pulling (Figure 6A). The left main stent was deployed in a poor position with an irretrievable deflated winged balloon inside it, as previously described. The balloon was partially entrapped between the surgical bioprosthesis post and the EDWARDS SAPIEN 3 frame.

As stated above, the ruptured balloon catheter shaft containing a few centimeters of a short segment remained attached to the entrapped balloon. The ruptured balloon catheter shaft extended a few centimeters into the aortic root. A goose neck snare was successfully used to retrieve the remaining proximal piece of the ruptured balloon catheter shaft out of the aortic root. A pulling force was again applied on the snare to facilitate the passage and retrieval of the entrapped balloon in-between the surgical post and the SAPIEN 3 frame. A second rupture occurred just outside of the surgical post and SAPIEN 3 frame, resulting in no movement of the entrapped deflated winged balloon (Figure 6B).

Access was lost to the partially, yet firmly, entrapped balloon inside the left main stent and in-between the surgical frame post and the SAPIEN 3 frame with no wire access. Attempting to wire the left main stent through a superior strut and next to the entrapped balloon was discussed. The strut was dilatated and another stent was deployed. This caused crushing of the entrapped balloon between two layers of the left main stent with the second stent extending further out in a vertical chimney fashion. This granted easy access to the left coronary system. Due to various reasons the left main stent was unable to be wired through a superior stent strut. This was due to the long distance between the guide catheter and the low left main coronary stent. The guide catheter 

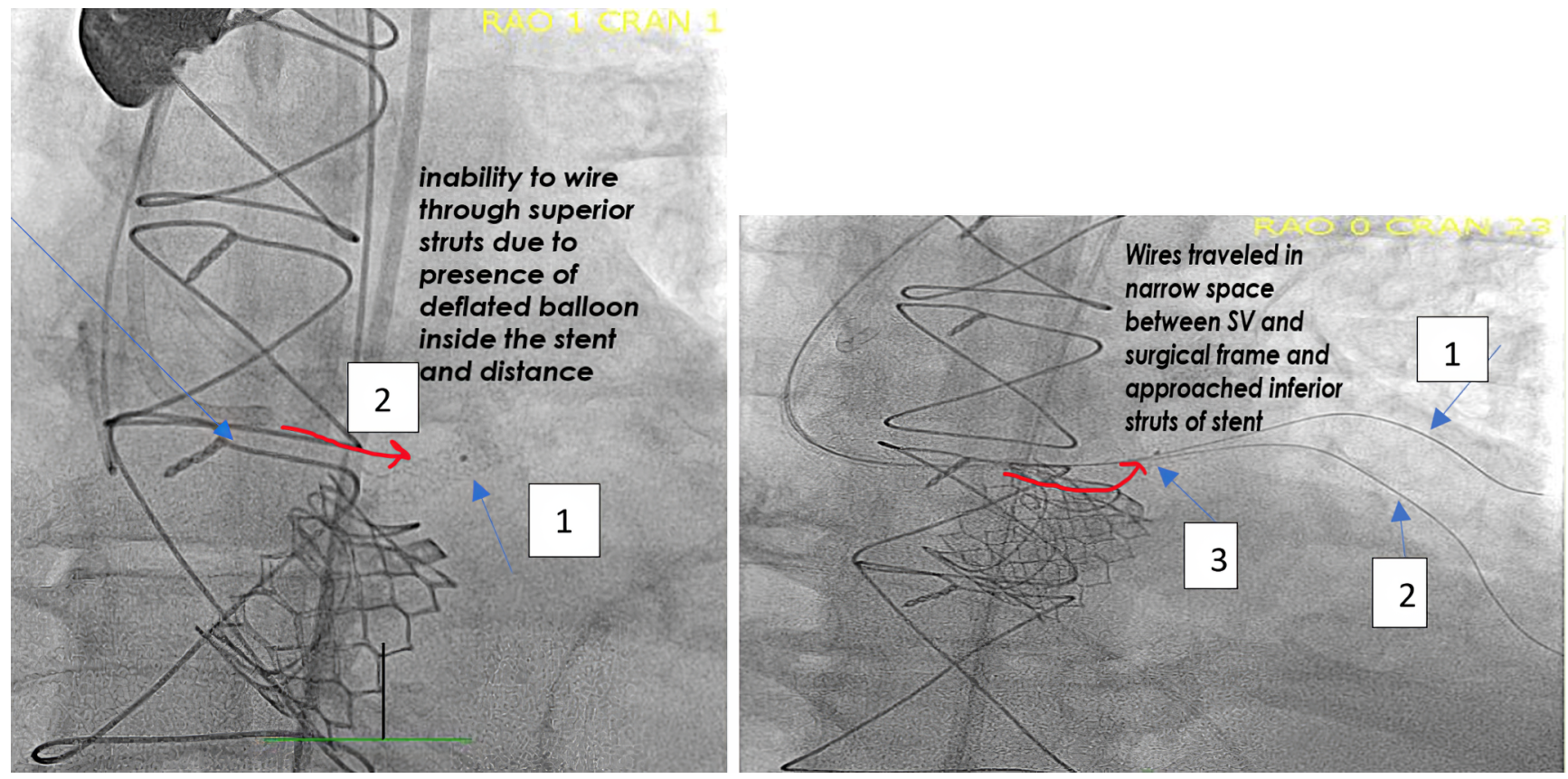

Figure 7. Panel $A$. Inability to wire through a superior strut due to distance and due to the presence of deflated winged balloon inside the stent. (A1) distal marker of stent balloon (A2) Coronary stent with deflated balloon entrapped inside. Panel B. Successfully wiring the stent through an inferior strut THV. (B1) wire \#1 (B2) wire \#2 introduced for better support (B3) distal marker of stent balloon.

was unable to advance further due to a narrow sinotubular junction and short VTC distance. Presence of the deflated winged balloon in the lumen of the left main coronary stent prohibited wire advancement (Figure 7A).

The patient remained hemodynamically stable with no electrocardiogram (ECG) changes during the procedure. The left main stent could not be wired from a superior strut with a deflated winged balloon as planned. The left main stent was wired through an inferior strut hoping for a more permissible wire advancement course next to the winged balloon. The hydrophilic wire with two curves at its tip was reshaped. A primary larger curve and a secondary smaller curve was used to advance the wire freely in the narrow distance between the surgical frame and the sinotubular junction. This allowed the hydrophilic wire to go inferior to the stent frame and redirected the wire more superiorly to engage the left main stent from below, through an inferior strut. After significant attempts the wire was advanced and positioned in the apical LAD successfully. The inferior entry strut was dilated with a $2.0 \mathrm{~mm}$ balloon and then a $2.5 \mathrm{~mm}$ balloon. The same maneuver was used to advance a second wire for better guide catheter support and to secure access to the left coronary arteries (Figure 7B).

The only option left was to advance a snare catheter distally to the deflated balloon while also attempting to snare the balloon from the distal end in the proximal LAD. An attempt was made to retrieve the deflated balloon backwards, forcing it to make a short U-turn in the proximal lumen of the LAD. This would allow the dilated inferior left main strut to be removed without needing to pass in-between the surgical and the SAPIEN 3 frames. The distal end of the balloon was successfully removed by positioning a snare in the mid LAD and gradually pulling it back to capture the distal nose of the deflated balloon. A forceful pull was again applied, successfully retrieving the deflated balloon outside the left main stent (Figure 8).

The existing left main stent will have a high risk of thrombosis due to its malposition as described above. One way to minimize this risk was to reattempt to wire the left main stent from a superior strut now that the deflated winged balloon was removed and no longer in the way. 


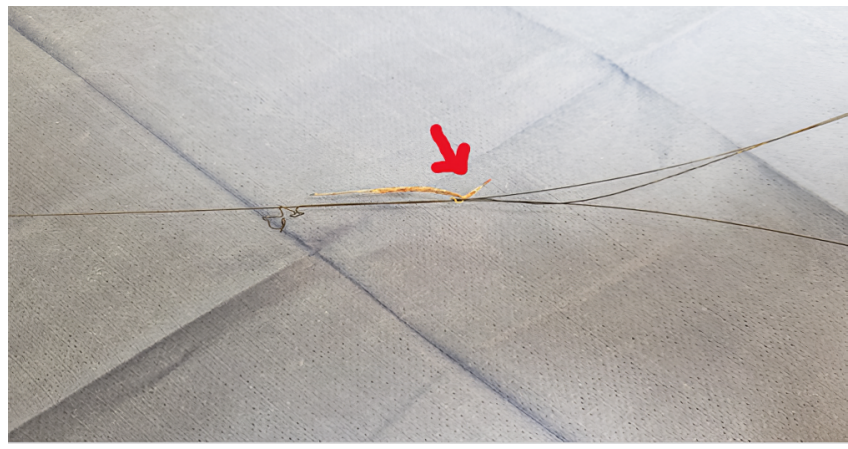

Figure 8. Stent balloon successfully snared and retrieved out of body.

The stent was successfully wired, dilating the superior entry strut. A $4.0 \mathrm{~mm}$ by $26 \mathrm{~mm}$ long stent extending vertically to the THV/SAPIEN 3 frames was deployed at the level of the sinotubular junction. This was followed by intravascular ultrasound (IVUS) evaluation, which revealed that the stent was undersized. The stent with a $5.0 \mathrm{~mm}$ non-compliant balloon was dilated and the procedure ended with a kissing left main. Balloon aortic valvuloplasty was performed using a $5.0 \mathrm{~mm}$ coronary balloon and a $24 \mathrm{~mm}$ Z-MED balloon. This concluded with an excellent angiographic result (Figure 9). A year later the patient was seen in the office with no recurrent admissions to the

hospital or any cardiac events. Mean gradient across the THV valve was $14 \mathrm{mmHg}$ following one year after the procedure.

\section{Discussion}

Transcatheter aortic valve replacement procedures are gaining more popularity with expanding indications. TAVR is already an approved indication for a failing surgical valve. Even though the TAVR procedure has become easier to perform with less complication rates, what remains critical is for the operators to be able to do a thorough analysis of the anatomy. In the case of the VIV procedure, both the structure of the surgical valve and the THV valve must be understood. One must have the ability to predict a complication and have a plan to manage it critically. Unpredicted complications can occur, such as the one reported in this case.

Retrospectively, the images reviewed at the end of the procedure showed an interaction occurred. This occurred during the inflation of the SAPIEN 3 stent valve balloon between the expanding SAPIEN 3 frame and the coronary stent catheter shaft. If noted during the procedure, it could have alerted to the fact that the coronary stent catheter shaft was coursing in-be-
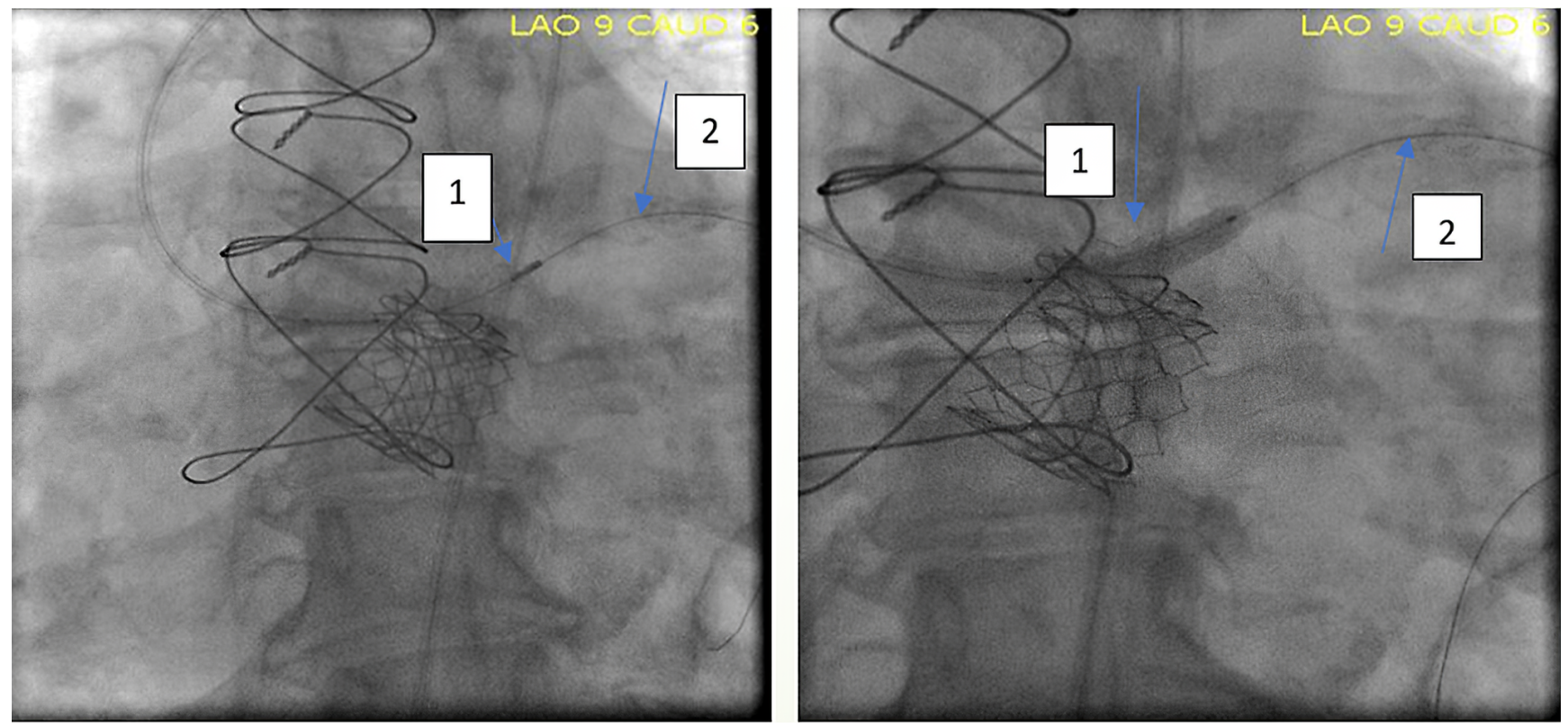

Figure 9. Panel $A$. (A1) IVUS catheter in L main stent. (A2) Wire through superior strut of left main stent. Panel B. (B1) Second left main stent creating a chimney path course through a superior strut of the first stent. (B2) Wire through a superior strut of the first left main stent. 
tween the surgical frame and the SAPIEN 3 frame. A quick adjustment or pull on the coronary stent catheter shaft could have been made to avoid the entrapment. It is advised to use the following for valve-invalve TAVR with high occlusion risk where balloon expandable valves are used. Close attention must be paid to the course of guide catheters, wires and coronary stent catheters to avoid entrapment. Monitoring for any interaction between the coronary stent catheter and the expanding THV frame during deployment as any interaction should indicate the coronary stent catheter is coursing in-between the THV and the surgical frames. Careful selection of guide catheters is important, such as using a Judkins left or short tip artery access as previously noted. Using general anesthesia with transesophageal echocardiogram (TEE) guidance allows for the use of smaller sized balloon expandable valves. Slight underfilling and lower deployment is desired as the end result.

\section{Conclusion}

Transcatheter aortic valve replacement is gradually becoming the main stream treatment for aortic valve stenosis. The skills to perform an interventional procedure are important, but more knowledge and training are indicated to perform the procedure successfully. Operators need to be able to perform careful analysis of the diagnostic data available to them and be able to predict potential risks and complications. Operators also need to be proficient in the various devices commercially available to them. Every operator must be ready to manage complications and be aware that potential unknown difficulties can occur. There is always a first time for everything.

\section{Conflict of Interest}

Safwan Kassas recieved a research grant from Edwards Life Science: Proctor Boston Scientific Inc.

\section{Comment on this Article or Ask a Question}

\section{References}

1. Hamid NB, Khalique OK, Monaghan MJ, Kodali SK, Dvir D, Bapat VN, et al. Transcatheter Valve Implantation in Failed Surgically Inserted Bioprosthesis: Review and Practical Guide to Echocardiographic Imaging in Valve-in-Valve Procedures. JACC Cardiovasc Imaging. 2015;8:960-979. DOI: https:// doi.org/10.1016/j.jcmg.2015.01.024

2. Dvir D, Leipsic J, Blanke $P$, Ribeiro $H B$, Kornowski R, Pichard A, et al. Coronary Obstruction in Transcatheter Aortic Valvein-Valve Implantation Preprocedural Evaluation, Device Selection, Protection, and Treatment. Circ Cardiovasc Interv. 2015;8:1. pii: e002079. DOI: https://doi.org/10.1161/ CIRCINTERVENTIONS.114.002079
3. Khan JM, Dvir D, Greenbaum AB, Babaliaros VC, Rogers T, Aldea G, et al. Transcatheter Laceration of Aortic Leaflets to Prevent Coronary Obstruction During Transcatheter Aortic Valve Replacement: Concept to First-in-Human. Circ Cardiovasc Interv. 2018;11:677-689. DOI: https://doi.org/10.1161/CIRCINTERVENTIONS.114.002079
Cite this article as: Kassas $S$, Fattal $P$, Sharma M. Entrapped Stent Delivery Catheter Shaft After High Risk TAVI: Retrieval \& Lessons Learned. Structural Heart Disease. 2019;5(5):229236. DOI: https://doi.org/10.12945/j. jshd.2019.037.18 